\title{
Effect of almond-enriched high-monounsaturated fat diet on selected markers of inflammation: a randomised, controlled, crossover study
}

\author{
Sujatha Rajaram*, Kristianne M. Connell and Joan Sabaté \\ Department of Nutrition, School of Public Health, Loma Linda University, Loma Linda, CA 92350, USA
}

(Received 14 July 2009 - Revised 10 September 2009 - Accepted 19 September 2009 - First published online 29 October 2009)

Frequent consumption of nuts lowers the risk of CHD. While lowering blood lipids is one of the mechanisms for cardioprotection, the present study sought to determine whether monounsaturated fat-rich almonds also influence other CHD risk factors such as inflammation and haemostasis. This was a randomised, controlled, crossover feeding study with twenty-five healthy adults (eleven men; fourteen women), age 22-53 years. Following a 2 week run-in phase ( $34 \%$ energy from fat), subjects were assigned in random order to three diets for 4 weeks each: a heart-healthy control diet with no nuts $(<30 \%$ energy from fat), low-almond diet and high-almond diet (10\% or $20 \%$ isoenergetic replacement of control diet with almonds, respectively). Serum $E$-selectin was significantly lower on the high-almond diet compared with the control diet. $E$-selectin decreased as the percentage of energy from almonds increased $(P<0.0001)$. C-reactive protein $(\mathrm{CRP})$ was lower in both the almond diets compared with the control diet. A clear dose response was not observed for either $E$-selectin or CRP. There was no effect of diet on IL- 6 or fibrinogen. Tissue plasminogen activator antigen was significantly lower on the control and high-almond diets compared with the low-almond diet, although the values were within normal range. In conclusion, consumption of almonds influenced a few but not all of the markers of inflammation and haemostasis. A clear dose response was not observed for any of the markers studied.

Monounsaturated fat: Almonds: Inflammation: Haemostasis: Endothelial dysfunction: CHD

Inflammation is becoming increasingly acknowledged as having a major role in the pathogenesis of $\mathrm{CHD}^{(1)}$ and certain cancers $^{(2)}$. Atherosclerosis may involve subclinical thrombosis and inflammation that can result in the activation of the haemostatic process ${ }^{(1,3)}$. Many dietary components ${ }^{(4)}$ have been shown to reduce the risk of CHD by mechanisms involving the lowering of blood lipids. One such component is nuts ${ }^{(5)}$ that are now being recommended as part of heart-healthy diets $^{(4)}$. Clinical studies in healthy and hypercholesterolaemic adults $^{(6,7)}$ have shown that nuts lower LDL-cholesterol while improving the overall blood lipid profile. In these studies, the cholesterol-lowering effects alone do not fully explain the percent decrease in CHD risk. Mechanisms beyond blood lipid lowering may also be contributing to the overall CHD risk reduction. Frequent nut and seed consumption is associated with lower levels of inflammatory markers such as C-reactive protein (CRP), IL-6 and fibrinogen, even after adjusting for confounding factors ${ }^{(8)}$, suggesting yet another mechanism by which nuts may reduce risk of CHD.

Nuts are very high in unsaturated fat and contain non-lipid components such as antioxidant vitamins (vitamin E), plant protein rich in arginine, $\mathrm{Mg}$, fibre and several phytochemicals $^{(9)}$. While the role of nuts high in monounsaturated fat such as almonds has not been previously studied in relation to inflammation, some of the components of nuts such as arginine, $\mathrm{Mg}$, fibre and vitamin $\mathrm{E}$ have demonstrated anti-inflammatory properties ${ }^{(5,10,11)}$. Recently, a portfolio diet consisting of cholesterol-lowering foods, including almonds, has showed a similar reduction in CRP as observed with statin therapy ${ }^{(12)}$. Similarly, a Mediterranean diet with nuts was effective in lowering IL-6 levels compared with a control diet without nuts ${ }^{(13)}$. In both these studies, nuts were one of the many components of the intervention diet, making it difficult to tease out the actual effect of nuts on inflammation. However, based on the nutrient and non-nutrient composition of nuts, one can hypothesise a favourable role for nuts in reducing inflammation.

The aim of the present study was to determine the influence, if any, of selected markers of inflammation in healthy adults. We also looked at a possible dose-response effect of almonds on these markers.

\section{Experimental methods}

\section{Study design}

The present study was part of an intervention trial ${ }^{(14)}$ designed to evaluate the effect of different doses of almonds on CHD risk factors. This was a randomised, single-blind, crossover, controlled feeding study. All subjects completed a 2-week run-in period during which they consumed a typical Western diet (34\% energy from fat). Following this, they were randomised to a sequence of three isoenergetic treatment

Abbreviations: CRP, C-reactive protein; tPA, tissue plasminogen activator.

* Corresponding author: Sujatha Rajaram, fax +1 909558 4095, email srajaram@1lu.edu 
diets for 4 weeks each: a cholesterol-lowering control diet without nuts ( $<30 \%$ energy from fat), a low-almond diet and a high-almond diet. The almond diets were the same as the control diet, but had 10 or $20 \%$ of the total energy replaced with almonds. This accounted for $34 \mathrm{~g} / 8368 \mathrm{~kJ}$ (2000 kcal) diet for the low-almond diet and $68 \mathrm{~g} / 8368 \mathrm{~kJ}$ $(2000 \mathrm{kcal})$ diet for the high-almond diet.

All meals were prepared and served in the Nutrition Department Research Kitchen at Loma Linda University. Sunday to Friday, breakfast and dinner were served on site, while lunch and all Saturday meals were packed to go. Nine menus were used in rotation to provide variety and the meals consisted of foods commonly consumed and represented all the food groups. Almonds were served on their own or incorporated into certain hot (e.g. pizza) or cold foods (e.g. salads). Menus were developed for seven levels of energy intake ranging from 7533 to $15066 \mathrm{~kJ}(1800-3600 \mathrm{kcal}) / \mathrm{d}$. Subjects were weighed every day during the run-in phase to establish their baseline body weight and then twice a week thereafter throughout the treatment phases, and their energy intake was adjusted as needed to maintain stable body weight throughout the study.

To ensure compliance to the treatments, the almonds were served either during breakfast or dinner and were eaten under the supervision of a senior investigator. In addition, subjects maintained a diary in which they recorded any deviations from the study diet protocols and this was reviewed on a weekly basis by a senior investigator. To ensure that the actual diet composition matched that of the planned, a random sampling of the diets was analysed for macronutrient composition (Covance Laboratory, Madison, WI, USA). The present study was carried out with the highest degree of quality control with compliance of subjects to diet and study protocol close to $100 \%$. A more detailed description of the diet protocol is provided elsewhere ${ }^{(14)}$.

\section{Subjects}

Subjects were included in the study if they were between the ages of 20 and 60 (mean age 40.9 (SE 12.8)) years, healthy, and if their habitual diet before the study was close to the typical American diet. Subjects were excluded if they had any previous history of hypertension, arteriosclerosis or metabolic diseases, had any weight change 6 months before the study, had BMI $>30 \mathrm{~kg} / \mathrm{m}^{2}$, fasting serum cholesterol $<15$ th or $>90$ th percentile for age, sex and race, fasting TAG $>2.26 \mathrm{mmol} / \mathrm{l}(>200 \mathrm{mg} / \mathrm{dl})$, were chronically ill or taking medications, consumed nuts frequently ( $>2$ times/ week), had erratic exercise habits or were athletes that had rigorous exercise routine, were smokers or drank alcohol $>2$ times a week. Women with irregular menses or hormone use within the past 5 years (either hormone replacement for postmenopausal women or women on birth control pills) were excluded from the study as well. Subjects were asked to maintain the same exercise pattern throughout the study that was established at the start. Subjects were asked to record in a daily diary any deviations from the exercise routine that was established at baseline and an investigator reviewed this diary on a weekly basis to ensure compliance to the exercise routine.
Out of the twenty-seven subjects that started, twenty-five completed the trial. The sample size of twenty-five subjects had $90 \%$ power to detect differences of $10 \%$ in $E$-selectin, at a significance level of $P<0.05$. Subjects were screened using a detailed medical and general questionnaire, oneon-one interviews and group meetings. The present study was conducted according to the guidelines laid down in the Declaration of Helsinki, and all procedures involving human subjects were approved by the Ethics Committee of Loma Linda University. Written informed consent was obtained from all study subjects.

\section{Data collection and analyses}

Twelve hour fasting blood samples were collected at the end of the run-in period and at the end of each dietary period on two alternate days by standard venepuncture. Samples were drawn at the same time in the morning on each occasion (between 06.30 and 08.30 hours). Blood was collected into two vacutainers: one coated with citrate and the other without any anticoagulants. The blood was kept on ice for $15 \mathrm{~min}$ and then centrifuged at $1500 \mathrm{~g}$ for $15 \mathrm{~min}$ at $4^{\circ} \mathrm{C}$. The plasma and serum samples were separated, aliquoted and stored at $-80^{\circ} \mathrm{C}$, and all analyses were completed within 1 year of completing the study. The erythrocytes from the citrate vacutainer were separated and washed three times with $150 \mathrm{mmol} / \mathrm{l}$ $\mathrm{NaCl}$ at $800 \mathrm{~g}\left(15 \mathrm{~min}\right.$ at $\left.4^{\circ} \mathrm{C}\right)$ and the buffy coat was removed. The red cells were mixed with saline $(50 \%$ packed cell volume) and stored at $-80^{\circ} \mathrm{C}$ until analysed for fatty acid composition.

Serum CRP, E-selectin, IL-6, plasma fibrinogen and tissue plasminogen activator (tPA) antigen were analysed at the end of the study (Department of Pathology, University of Vermont, Burlington, VT, USA). These makers were selected from the many different markers available because they have been previously shown to be influenced by nuts or dietary fatty acids. Fibrinogen was measured in an automated clot-rate assay $^{(15)}$ using an ST4 instrument (Diagnostica Stago, Parsipanny, NJ, USA). Plasma tPA antigen was determined by a two-site immunoassay utilising three different monoclonal antibodies $^{(16)}$. This assay is sensitive to both free tPA and tPA in complex with its inhibitors. $E$-selectin and IL-6 were measured by ultra-sensitive ELISA (R\&D Systems, Minneapolis, MN, USA). A high-sensitivity colorimetric competitive immunoassay was used to determine CRP levels ${ }^{(17)}$. Serum and erythrocyte samples were also analysed for fatty acid composition (Lipomics, West Sacramento, CA, USA) using methods previously described in the present original study ${ }^{(14)}$.

\section{Statistical analysis}

Statistical analysis was performed with the Statistical Analysis System, version 8.0 (SAS Institute Inc., Cary, NC, USA). Duplicate measurements of the outcome variables were averaged and used in further analysis. Changes in inflammatory and haemostatic factors in response to dietary treatment were determined by ANOVA using mixed linear models that included a random-effect term for subjects and fixed-effect term for diet and period. Tests for trend were conducted by ANOVA by replacing the diet variables in the above model with continuous variable representing the percent total 
energy in the diet contributed by almonds. Results with a $P$ value of $\leq 0.05$ were considered statistically significant. Results are reported as the least-squares means with their standard error. Fibrinogen, IL-6 and CRP were found to violate the assumption of homogeneity of variance. Therefore, the log-transformed values were used for statistical analysis.

\section{Results}

Out of the twenty-seven subjects that started the study, two dropped out for lack of adherence to the study protocol and twenty-five completed all the requirements. Of those that completed eleven were women and fourteen were men. The mean age of the subjects was 41 (SE 13) years (range 22-53 years). Subjects were apparently healthy with normal baseline cholesterol and TAG levels (mean), baseline serum cholesterol $5 \cdot 61$ (SE 0.14) mmol/l, LDL cholesterol 3.86 (SE 0.15) mmol/1 and TAG 1.37 (SE 0.13) mmol/l. The mean baseline body weight of study subjects was 71 (SE 2.7) kg. Body weight remained constant throughout the study (control diet 71 (SE 2.7) kg; low-almond diet 71.2 (SE 2.7) kg; high-almond diet $70 \cdot 7$ (SE 2.7) kg).

Table 1 shows the analysed diet composition of the three diets. As expected, the percent energy from fat was higher in the low- $(35 \%)$ and high-almond diets $(39 \%)$ compared with the control diet $(30 \%)$. The incorporation of almonds in the diets resulted in small decreases in saturated fat, small increases in polyunsaturated fat and a substantial increase in monounsaturated fat.

The changes in the fatty acid composition of plasma TAG and erythrocyte membrane phospholipids during the treatment diets are reported in Table 2 . The percentage of total monounsaturated fat increased with increasing amounts of almonds in the diet both in the erythrocyte membrane and serum TAG lipid fraction. This trend is similar to those observed for monounsaturated fat content of the diets (Table 1), and validates the consumption of almonds and close adherence to the diets by the subjects.

The results of the almond diets on inflammatory markers are presented in Table 3. Differences between diet treatments showed that the high-almond diet had significantly lower $E$-selectin than the control $(7.8 \%$ decrease) and low-almond $(6 \cdot 3 \%$ lower) diets, but there were no differences between low-almond and control diets. For each $1 \%$ increase in energy from almonds, there was an estimated decrease of

Table 1. Macronutrient composition of the three diets*

\begin{tabular}{lccc}
\hline & Control & Low almond & High almond \\
\hline Nutrient & & & \\
Energy (kJ/d) & 10133 & 10401 & 10242 \\
Protein (\% energy) & 14.0 & 13.4 & 14.1 \\
Carbohydrate (\% energy) $\dagger$ & 55.8 & 51.2 & 46.0 \\
Fat (\% energy) & 29.9 & 35.0 & 39.0 \\
SFA (\% energy) & $8 \cdot 2$ & $8 \cdot 0$ & $7 \cdot 7$ \\
MUFA (\% energy) & $12 \cdot 1$ & 16.5 & 19.4 \\
PUFA (\% energy) & $6 \cdot 2$ & 7.5 & 8.7 \\
Cholesterol (mg/d) & 202 & 163 & 140 \\
\hline
\end{tabular}

*Values obtained during chemical analysis of samples from the study diets.

$\dagger$ The values for carbohydrate intake were calculated by subtracting the values for fat and protein intake from those of total energy intake.
$0 \cdot 18 \mu \mathrm{g} / \mathrm{l}$ in $E$-selectin. Serum $E$-selectin decreased with progressively higher amounts of almonds in the diet $(P<0.0001)$, but there was no clear dose response.

Both the almond-enriched diets lowered serum CRP compared with the control, diet but there was no dose response. For serum IL-6 $(P=0.64)$ and fibrinogen $(P=0.89)$, there were no treatment effects. For tPA, lowalmond diet had significantly higher values than highalmond and control diets $(P<0 \cdot 01)$.

\section{Discussion}

Frequent consumption of nuts is associated with a reduced risk of $\mathrm{CHD}^{(5)}$. One of the mechanisms by which they may bring about their cardioprotective effects is by lowering blood lipids. We had previously shown that $68 \mathrm{~g}$ almonds for $8386 \mathrm{~kJ}(2000 \mathrm{kcal})$ diet per day lowers serum total and LDL-cholesterol in healthy adults ${ }^{(14)}$. In the present study, we further explored the effects of an almond-enriched diet on markers of inflammation. We showed that consuming a high-almond diet $(68 \mathrm{~g} / \mathrm{d}$ per $8386 \mathrm{~kJ})$ for 4 weeks significantly decreased serum $E$-selectin compared with the isoenergetic control diet in healthy men and women. Increasing the percent energy from almonds and thus monounsaturated fat content in the diet progressively lowered $E$-selectin, but no clear dose response was observed. We showed that both the almond diets were able to lower serum CRP compared with the control diet but not in a dose-dependent manner. Other markers of inflammation and haemostasis that we studied were not influenced by the almond-enriched diets.

Circulating levels of adhesion molecules such as $E$-selectin are critical to leucocyte and platelet recruitment and are indicative of endothelial dysfunction ${ }^{(18)}$. A decrease in $E$-selectin could lower the inflammatory response and thus lower the risk of CHD. Our data suggest that the decrease in serum $E$-selectin and CRP in subjects during the highalmond diet could be explained by the change in MUFA concentration in the diets. This is in fact verified by a significant increase in both erythrocyte membrane and plasma TAG MUFA content in subjects during the high-almond diet compared with the control diet. It is possible that the effects of dietary fatty acids on $E$-selectin may be mediated by alteration in gene expression of these adhesion molecules ${ }^{(19)}$.

A study similar to ours, but using olive oil as the source of MUFA $^{(20)}$, reported no effect of a high-MUFA diet on plasma $E$-selectin levels. Although this was also a feeding study with the same duration of intervention as ours, there were a few differences that may have contributed to the discordant outcomes. The baseline plasma $E$-selectin concentration reported in their study was almost half that observed in ours, suggesting that the effect of MUFA is perhaps seen only at higher concentrations of $E$-selectin. Also, subjects in their study were men less than 30 years, while the present study had both men and women from 20 to 60 years. The sources of MUFA in the two studies were also different suggesting that other non-lipid bioactive components in almonds may have influenced $E$-selectin. Dietary polyphenols have been shown to modulate signal transduction pathways causing the repression of pro-inflammatory genes ${ }^{(21)}$. Almonds, especially eaten with their skin intact, have a significant amount of flavonoids that seem to help protect LDL from 
Table 2. Distribution of fatty acids in serum TAG and erythrocyte membrane phospholipids (mole \%) (Mean values with their standard errors)

\begin{tabular}{|c|c|c|c|c|c|c|c|}
\hline \multirow[b]{2}{*}{ Fatty acid } & \multicolumn{2}{|c|}{ Control } & \multicolumn{2}{|c|}{ Low almond } & \multicolumn{2}{|c|}{ High almond } & \multirow[b]{2}{*}{$P$ for trenc } \\
\hline & Mean & SE & Mean & $\mathrm{SE}$ & Mean & $\mathrm{SE}$ & \\
\hline \multicolumn{8}{|l|}{ Saturated fat } \\
\hline Serum & $33 \cdot 2$ & 0.7 & $33 \cdot 2$ & 0.7 & $30 \cdot 9$ & 0.7 & 0.01 \\
\hline Erythrocyte & 43.9 & 1.6 & 43.9 & 1.2 & $43 \cdot 27$ & 1.3 & NS \\
\hline \multicolumn{8}{|c|}{ Monounsaturated fat } \\
\hline Serum & $32 \cdot 7$ & 0.4 & 34.6 & 0.4 & $36 \cdot 3$ & 0.4 & $<0.001$ \\
\hline Erythrocyte & 18.5 & 1.5 & $18 \cdot 6$ & 1.3 & $19 \cdot 0$ & 1.3 & $<0.01$ \\
\hline \multicolumn{8}{|c|}{ Polyunsaturated fat } \\
\hline Serum & $21 \cdot 7$ & 0.1 & $20 \cdot 9$ & 0.1 & $22 \cdot 1$ & 0.1 & NS \\
\hline Erythrocyte & $37 \cdot 3$ & $3 \cdot 1$ & $37 \cdot 2$ & 3.0 & 37.5 & $2 \cdot 4$ & NS \\
\hline
\end{tabular}

oxidation $^{(22)}$, and whether it has a role in suppressing gene expression of adhesion molecules remains to be determined.

CRP is a sensitive marker of inflammation and high levels are strongly linked with coronary events, stroke and peripheral vascular disease ${ }^{(3)}$. There are only two other studies that have looked at the effects of almonds on serum CRP, one of which showed no change in CRP levels ${ }^{(23)}$ and the other showed a decrease in serum CRP levels with a portfolio diet that contained many cholesterol-lowering foods including almonds ${ }^{(12)}$. Because multiple dietary changes were made in the portfolio diet study, one cannot tease out the effects of almonds. Although the present study showed a decrease in serum CRP of about $4 \cdot 5-9 \%$ on almond diets, the mean individual values were in the moderate risk estimate $(1-3 \mathrm{mg} / \mathrm{l})$ at baseline and at the end of the diet period. Almonds are rich in $\mathrm{Mg}$, which have been inversely related to serum CRP levels ${ }^{(24)}$, and may be a potential mechanism that might explain the effects of almonds on CRP warranting further investigation. Also other bioactive components in almonds including arginine, $\alpha$-tocopherol and phytonutrients ${ }^{(9,10,25,26)}$ may have a role in modifying some of the inflammatory mediators, but more research is needed to fully understand their role.

While serum CRP levels were influenced by almonds, no significant change was observed for serum IL-6 although the direction of change was the same as for CRP. Serum IL-6 tends to be lower in the morning and higher at night, while CRP does not appear to go through similar diurnal variations $^{(27)}$. Therefore, CRP tends to be better able to predict CHD in comparison with IL- 6 on morning-extracted blood samples. The haemostasis factors examined in the present study were also not influenced by the almond-enriched diets. Plasma tPA antigen was significantly lower on the control and high-almond diets in comparison with the low-almond diet. However, the concentrations were at the lower end of the normal range, and the observed differences do not appear to be biologically significant. We found no effect of almonds on plasma fibrinogen and others have made similar observations $^{(28)}$ with dietary fatty acids.

Several things may explain the lack of an effect on these haemostatic factors including a small sample size, baseline values and duration of intervention. While a sample size of 25 was adequate to detect a $10 \%$ difference in inflammatory markers at $90 \%$ power, thirty-six subjects would be required for similar statistical power for haemostatic variables. Also, 4 weeks of intervention although sufficient to see changes in blood lipids ${ }^{(29)}$ and some selected inflammatory markers, it may not be adequate to measure changes in haemostatic factors. Finally, it is likely that neither MUFA nor other bioactive components in almonds influence haemostasis factors especially in an apparently healthy population with baseline values near lower end of normal. Studies suggest that in participants that already have elevated inflammatory status like in obesity, metabolic syndrome or cancer do respond more favourably to bioactive components in food ${ }^{(30-32)}$. The role of almonds and other nuts in reducing inflammation in such clinical populations needs to be explored in the future.

Tree nuts and peanuts are fatty food containing mostly unsaturated fatty acids. Of the different nuts, almonds,

Table 3. Inflammatory markers after the three treatment diets

(Mean values with their standard errors and standard deviations)

\begin{tabular}{|c|c|c|c|c|c|c|c|c|c|}
\hline \multirow[b]{2}{*}{ Markers } & \multicolumn{2}{|c|}{ Baseline* } & \multicolumn{2}{|c|}{ Control } & \multicolumn{2}{|c|}{ Low almond } & \multicolumn{2}{|c|}{ High almond } & \multirow[b]{2}{*}{$P$ for trend } \\
\hline & Mean & SD & Mean & SE & Mean & SE & Mean & SE & \\
\hline \multicolumn{10}{|l|}{ Inflammation } \\
\hline E-selectin $((\mathrm{g} / \mathrm{l})$ & 55.9 & $22 \cdot 9$ & $54 \cdot 3^{a}$ & $4 \cdot 6$ & $53 \cdot 5^{a}$ & $4 \cdot 6$ & $50 \cdot 1^{b}$ & 4.6 & $<0.0001$ \\
\hline $\mathrm{CRP}(\mathrm{mg} / \mathrm{l})$ & 1.53 & 1.52 & $1.54^{\mathrm{a}}$ & 0.31 & $1.40^{\mathrm{b}}$ & 0.31 & $1.47^{\mathrm{b}}$ & 0.31 & 0.03 \\
\hline IL-6 (ng/l) & $1 \cdot 18$ & 0.96 & $1 \cdot 39$ & 0.25 & $1 \cdot 25$ & 0.26 & 1.35 & 0.26 & 0.64 \\
\hline \multicolumn{10}{|l|}{ Haemostasis } \\
\hline Fibrinogen (g/l) & 3.0 & 0.5 & $2 \cdot 9$ & $0 \cdot 12$ & 3.0 & $0 \cdot 12$ & $2 \cdot 9$ & 0.12 & 0.89 \\
\hline tPA ag $((\mathrm{g} / \mathrm{l})$ & 1.23 & 1.27 & $1 \cdot 15^{\mathrm{a}}$ & 0.18 & $1.29^{b}$ & 0.18 & $1 \cdot 12^{\mathrm{a}}$ & 0.18 & 0.71 \\
\hline
\end{tabular}

CRP, C-reactive protein; tPA ag, tissue plasminogen activator antigen.

${ }^{a, b}$ Mean values with different superscript letters were significantly different by ANOVA $(P<0.05)$

${ }^{*}$ Baseline values were measured at end of the run-in phase. 
peanuts, pistachio, macadamia and pecans are some of the monounsaturated fat-rich nuts, while walnuts are rich in polyunsaturated fat ${ }^{(9)}$. The role of nuts in decreasing inflammation has received more attention recently with the Multi-Ethnic Study of Atherosclerosis first showing an inverse association between frequent consumption of nuts and seeds and serum CRP, IL-6 and fibrinogen levels ${ }^{(8)}$. Two other studies showed that a Mediterranean diet pattern, which includes nuts, is associated with anti-inflammatory markers more strongly than diets with no nuts ${ }^{(33,34)}$. While nuts share a similar fatty acid profile, they do constitute different bioactive components that make up their non-lipid matrix ${ }^{(9)}$. Therefore, whether or not all nuts produce similar changes in markers of inflammation needs to be explored in future studies. Studies to date have mainly looked at walnuts ${ }^{(35,36)}$ and almonds $s^{(12,23)}$ with respect to inflammation.

The present study shows that incorporating about $68 \mathrm{~g}$ almonds per $8368 \mathrm{~kJ}(2000 \mathrm{kcal})$ cholesterol-lowering diet decreases serum $E$-selectin and CRP in healthy men and women, but does not influence other markers like IL-6 and haemostasis factors. There is some evidence to suggest that even small changes in CRP observed within the normal range are predictive of vascular events in healthy individuals $^{(37)}$. While the absolute change observed in some of the markers may be small, the additive effect of different markers on CHD risk reduction may be more meaningful clinically. Further studies are therefore needed to substantiate the present findings, elucidate mechanisms of action and determine the biological and clinical significance of these diet-induced changes to the overall disease process. While the evidence supporting the inclusion of nuts in heart-healthy diets is well established, it is being increasingly recognised that nuts provide cardiovascular protection that go beyond cholesterol lowering.

\section{Acknowledgements}

The funding for the present research was provided by the Center for Health and Nutrition Research at the University of California, Davis, and by the Almond Board of California, Fresno, CA. We acknowledge the support provided by Dr Russell Tracy, University of Vermont, Burlington, VT, USA for analysing many of the serum markers in the present study. The authors have no conflicts of interest.

S. R. and J. S. designed the study. S. R. coordinated the study. S. R. and K. M. C. were responsible for data collection and analyses and quality control. K. M. C. and J. S. were involved in statistical analyses. K. M. C. was the dietitian and was responsible for all diet analyses. All authors contributed to the interpretation of data. S. R. and K. M. C. wrote the first draft of the manuscript and all authors critically reviewed and revised the manuscript. S. R. obtained the funding for the present study.

\section{References}

1. Hanson GK (2005) Inflammation, atherosclerois, and coronary artery disease. $N$ Engl J Med 352, 1685-1695.

2. Marx J (2004) Inflammation and cancer: the link grows stronger. Sci Tech Froid 306, 966-968.
3. Tedgui A \& Mallat Z (2006) Cytokines in atherosclerosis: pathogenic and regulatory pathways. Physiol Rev 86, 515-581.

4. Lichtenstein AH, Appel LJ, Brands M, et al. (2006) Diet and lifestyle recommendations revision 2006: a scientific statement from the American Heart Association Nutrition Committee. Circulation 114, 82-96.

5. Kris-Etherton PM, Hu FB, Ros E, et al. (2008) The role of tree nuts and peanuts in the prevention of coronary heart disease: multiple potential mechanisms. J Nutr 138, 1746S-1751S.

6. Mukuddem-Petersen J, Oosthuizen W \& Jerling J (2005) A systematic review of the effects of nuts on blood lipid profiles in humans. J Nutr 135, 2082-2089.

7. Griel AE \& Kris-Ehterton PM (2006) Tree nuts and the lipid profile: a review of clinical studies. Br J Nutr 96, S68-S78.

8. Jiang R, Jacobs DR Jr, Mayer-Davis E, et al. (2006) Nut and seed consumption and inflammatory markers in the MultiEthnic Study of Atherosclerosis. Am J Epidemiol 163, 222-231.

9. Ros E (2008) Nuts: consumption, composition, health benefits and safety. $C A B \operatorname{Rev} 3,1-12$.

10. Blomhoff R, Carlsen MH, Frost AL, et al. (2006) Health benefits of nuts: potential role of antioxidants. Br J Nutr 96, S52-S60.

11. Wells BJ, Mainous AG \& Everett CJ (2005) Association between dietay arginine and C-reactive protein. Nutrition 21, 125-130.

12. Jenkins DJA, Kendall CWC, Marchie A, et al. (2005) Direct comparison of dietary potfolio vs. statin on C-reactive protein. Eur J Clin Nutr 59, 851-860.

13. Estruch R, Martinez-González MA, Corella D, et al. (2006) Effects of a Mediterranean-style diet on cardiovascular risk factors: a randomized trial. Ann Intern Med 145, 1-11.

14. Sabaté J, Haddad E, Tanzman J, et al. (2003) Serum lipid response to the graduated enrichment of a step I diet with almonds: a randomized feeding trial. Am J Clin Nutr 77, 1379-1384.

15. Clauss A (1957) A Gerrinnungs physiologische schnell Methode zur Bestimmung des Fibrinogens. Acta Haematol 17, 237.

16. Holvoet P, Cleemput H \& Collen D (1985) Assay of human tissue-type plasminogen activator [t-PA] with an enzymelinked immunosorbent assay [ELISA] based on three murine monoclonal antibodies to t-PA. Thromb Haemostas 54, 684-697.

17. Macy E, Hayes T \& Tracy R (1997) Variability in the measurement of C-reactive protein in healthy subjects: implication for reference interval and epidemiological applications. Clin Chem 43, 52-58.

18. Bonetti PO, Lerman LO \& Lerman A (2003) Endothelial dysfunction: a marker of atherosclerotic risk. Thromb Vasc Biol 23, $168-175$.

19. Bellido C, López-Miranda J, Pérez-Martinez P, et al. (2006) The Mediterranean and CHO diets decrease VCAM-1 and E-selectin expression induced by modified low-density lipoprotein in HUVECs. Nutr Metab Cardiovasc Dis 16, 524-530.

20. Perez-Jimenez F, Castro P, Lopez-Miranda J, et al. (1999) Circulating levels of endothelial function are modulated by dietary monounsaturated fat. Atherosclerosis 145, 351-358.

21. Rahman I, Biswas SK \& Kirkham PA (2006) Regulation of inflammation and redox signaling by dietary polyphenols. Biochem Pharmacol 72, 1439-1452.

22. Chen CY, Milbury PE, Chung SK, et al. (2007) Effect of almond skin polyphenolics and quercetin on human LDL and apolipoprotein B-100 oxidation and conformation. Nutr Biochem 18, 785-794.

23. Jenkins DJA, Kendall CW, Marchie A, et al. (2002) Dose response of almonds on coronary heart disease risk factors: blood lipids, oxidized low-density lipoproteins, lipoprotein(a), homocysteine, and pulmonary nitric oxide: a randomized, controlled, crossover trial. Circulation 106, 1327-1332. 
24. Song Y, Li TY, van Dam RM, et al. (2007) Magnesium intake and plasma concentrations of markers of systemic inflammation and endothelial dysfunction in women. Am J Clin Nutr 85, $1068-1074$.

25. Huynh NN \& Chin-Dusting $\mathbf{J}$ (2006) Amino acids, arginase and nitric oxide in vascular health. Clin Exp Pharmacol Physiol 33, $1-8$.

26. Jambazian PR, Haddad E, Rajaram S, et al. (2005) Almonds in the diet simultaneously improve plasma alpha-tocopherol concentrations and reduce plasma lipids. J Am Diet Assoc 105, 449-454.

27. Meier-Ewert HD, Ridker PM, Rifai N, et al. (2001) Absence of diurnal variation of $\mathrm{C}$-reactive protein concentrations in healthy human subjects. Clin Chem 47, 426-430.

28. Temme EH, Mensink RP \& Hornstra G (1999) Effects of diets enriched in lauric, palmitic or oleic acids on blood coagulation and fibroinolysis. Throb Haemost 81, 259-263.

29. Kris-Etherton PM \& Dietschy J (1997) Design criteria for studies examining individual fatty acid effects on coronary heart disease risk factors: human and animal studies. Am J Clin Nutr 65, 1590S-1596S.

30. Browning LM, Krebs JD, Moore CS, et al. (2007) The impact of long chain $n$-3 poluunsaturated fatty acid supplementation on inflammation, insulin sensitivity and CVD risk in a group of overweight women with an inflammatory phenotype. Diabetes Obes Metab 9, 70-80.
31. Esposito K, Marfella R, Ciotola M, et al. (2004) Effect of a Mediterranean-style diet on endothelial dysfunction and markers of vascular inflammation in the metabolic syndrome: a randomized trial. J Am Med Assoc 292, 1440-1446.

32. Kim YS, Young MR, Bobe G, et al. (2009) Bioactive food components, inflammatory targets, and cancer prevention. Cancer Prev Res (Phila Pa) 2, 200-208.

33. Montzoros CS, Williams CJ, Manson JE, et al. (2006) Adherence to the Mediterranean dietary pattern is positively associated with plasma adiponectin concentration in diabetic women. Am J Clin Nutr 84, 328-335.

34. Salas-Salvado J, Garcia-Arellano A, Estruch R, et al. (2008) Components of the Mediterranean-type food pattern and serum inflammatory markers among patients at high risk for coronary heart disease. Eur J Clin Nutr 62, 651-659.

35. Ros E, Nunez I, Perez-Heras A, et al. (2004) A walnut diet improves endothelial function in hypercholesterolemic subjects: a randomized crossover trial. Circulation 109, $1609-1614$.

36. Mukuddem-Petersen J, Stonehouse Oosthuizen W, Jerling JC, et al. (2007) Effects of a high walnut and high cashew nut diet on selected markers of the metabolic syndrome: a controlled feeding trial. Br J Nutr 97, 1144-1153.

37. Hackam DC \& Anand SS (2003) Emerging risk factors for atherosclerotic vascular disease: a critical review of the evidence. J Am Med Assoc 290, 932-940. 